\title{
Not every peer is a Peer
}

Letztes Jahr las ich einen kurzen (deutschen) Beitrag, in dem der Ursprung des Wortes und Begriffes „peer review“ erklärt wurde. Da hieß es, dass der Ursprung auf die Royal Society of London zurückgeht, die mit den Philosophical Transactions 1657 die erste wissenschaftliche Zeitschrift eingeführt habe. Mitglieder des englischen Oberhauses, die Peers, hätten, so hieß es, den Autoren Schutz vor Plagiaten und geistigem Diebstahl garantiert. Sie hätten im Gegenzug gefordert, dass die Arbeiten begutachtet werden, und dass sich die Autoren dem Urteil der Gutachter beugen. So sei es zu dem Ausdruck „peer review“ gekommen.

Eine nette Geschichte, aber leider stimmt sie nicht; doch ist sie gut erfunden - von wem auch immer. Die Royal Society of London for the Improvement of Natural Knowledge (so hieß sie genau) wurde 1660 gegründet, unter Obhut des Königs Charles II (daher „Royal“), von Wissenschaftlern wie Boyle, auch Newton und Wren (keiner zu der Zeit ein Lord). Die Philosophical Transactions wurden 1665/1666 gegründet. Nirgends ist zu finden, dass das House of Lords irgendetwas garantiert hätte, zumal unter Cromwell das House abgeschafft war und erst nach der Restoration 1660 wieder eröffnet wurde.

Zu jener Zeit gab es auch noch gar nicht den Begriff des Plagiats wissenschaftlicher Arbeiten. Aber noch wichtiger ist, daran zu erinnern, dass fast alle Zeitschriften, bis in das 20. Jahrhundert, von Herausgebern bzw. Schriftleitern ganz autokratisch geführt wurden. Nur manchmal fragten sie andere nach ihrer Meinung. In den Philosophical Transactions wurde publiziert, was vorgetragen wurde.

In Wirklichkeit hat der Begriff peer weder mit der Royal Society noch mit den Lords irgendetwas zu tun. Er hat einen gutbürgerlichen Anfang: Das Wort peer bedeutet nämlich „gleich“, wie z.B. in „gleich gestellt“ oder „diese beiden sind sich gleich“. Das Wort, bereits vor dem 14. Jahrhundert im Gebrauch, hat seinen Ursprung wahrscheinlich, mit vielen späteren etymologischen Umänderungen, in dem lateinischen Wort par-um=gleich. Als „sich gleichstehende“ wurde dieses Wort in Bezug auf die Mitglieder des House of Lords übernommen und so kam es zum Peer mit dem großen P. Aber peer ist eigentlich erst in den letzten 50 Jahren populär geworden, vor allem unter Soziologen und Psychologen (z.B. „peer group; peer response; he relates well to his peers").

Wie erwähnt, vor vielen Jahren gab es Herausgeber von medizinisch-wissenschaftlichen Zeitschriften, die alles was Veröffentlichung anbetraf, selbst entschieden (manchen gehörte die Zeitschrift sogar). Es waren immer nur Herren, und viele gebaren sich wie (zuweilen auch gutartige) Herrscher. Auch Herausgeber gab es und mit wachsender Spezialisierung wurden der eine oder andere Beitrag an Sachkundige zur Beurteilung geschickt. Als aber mit logarithmisch ansteigender Zahl von immer enger umschriebenen Spezialgebieten es schwer fiel, Arbeiten inhaltlich gut zu beurteilen und zu bewerten, musste man sich an externe Experten im selben Gebiet wenden (also meistens solche, die mehr und mehr über immer engere Gebiete wussten). Sie sollten dem Autor im Wissen gleich stehen, d.h. man hat deren peers geholt, zum peer review (zur Beurteilung bzw. Überprüfung). Aber auch dieser Weg, so hat sich inzwischen gezeigt, geht manchmal schief.

Fragen sind aufgekommen: Wer wählt wen zur Beurteilung, sollten Beiträge anonym beurteilt werden, wer ist tatsächlich „verantwortlich“ für Entscheidungen, wie kann man persönliche Vorurteile, idiosynkratisches Denken (usw.) verhindern? So treffen sich regelmäßig Schriftleiter, Verleger, Mitarbeiter, Journalisten, Rechtsanwälte und manche andere zu einem internationalen „Meinungsaustausch“, um Probleme des peer review zu diskutieren und (wer mag's glauben?) zu lösen. Vorläufig bleibt es aber beim peer review, anonym oder nicht, bei solchen wissenschaftlichen wie auch medizinischen Zeitschriften, die ernst genommen werden wollen. Veröffentlichungen in Zeitschriften, deren Beiträge nicht dem peer review unterliegen, werden 
oft in wissenschaftlichen Kreisen (berechtigt oder unberechtigt) nicht ganz anerkannt.

Die Einführung des peer review hat zweifellos einige weitere (nicht ganz so ernst gemeinte) Vorteile gebracht: 1. Es ermöglicht Schriftleitern und Beiratsmitgliedern wissenschaftlicher (einschließlich medizinischer) Zeitschriften, ohne kugelsichere Weste an Kongressen teilzunehmen, selbst wenn sie von einem „abgelehnten“ Autor erkannt werden sollten. 2. Bei Stellenbewerbung kann man „Objektivierung“ erreichen, indem nur Veröffentlichungen aus peer review Zeitschriften bewertet werden, nicht der früher allgemein beliebte GPJ Index (Gewicht aller Publikationen pro Jahr). 3. Durch sorgfältige Bespitzelung kann ein abgelehnter Autor herausfinden, wer den Wahn hat zu glauben, sein peer zu sein. 4. Nach Ablehnung einer dem Autoren sehr wichtig erscheinenden Arbeit kann er mit Freude und Überzeugung anderen stolz sagen, dass es offensichtlich keinen ihm gleichgestellten Berater gegeben hat.

Letztlich veröffentlichen schon viele wissenschaftlichen Zeitschriften gewisse Beiträge auf ihrer website über das Internet, oft ohne peer review und vor einer eventuellen „offiziellen“ Veröffentlichung im Heft, um sie schnell zu bringen. Große Institutionen entwickeln eigene web sites um „in-house“ Forschung schnellstens der Welt zu zeigen. Es erinnert etwas an die nicht allgemein bekannte Situation vor fast 50 Jahren, kurz nach der „double helix“ Entdeckung von Crick und Watson. Danach häuften sich Arbeiten über die DNA derart, dass die (damals verhältnismäßig wenigen) Wissenschaftler in der neuen molekularen Biologie sich mittels der damals gerade eingeführte FacsimileÜbertragung informierten, denn man konnte nicht warten, bis etwas publiziert wurde.

Manche englisch-sprachigen medizinischen Zeitschriften lassen (wie auch die DMW) nicht nur Originalien, sondern auch Beiträge anderer Rubriken (z.B. Übersichten) begutachten, schon allein, um einseitige oder selektive Darstellung zu vermeiden. Aber Vorurteile, Eitelkeiten und Idiosynkratien gibt es ja auf beiden Seiten, bei Autoren wie Gutachtern. Diese Bedenken, wie andere mehr prinzipieller Art, haben dazu geführt, dass einige Zeitschriften auf Anonymität des peer review (Begutachter und Autoren, einschließlich der Institution, sind gegenseitig unbekannt) bestehen.
Allerdings haben vergleichende, „blinde“ Untersuchungen meistens keinen signifikanten Unterschied gefunden. Dabei muss berücksichtigt werden, dass manche potenzielle Begutachter es ablehnen, ihre Anonymität - aus welchen Gründen auch - aufzugeben. Letzten Endes muss es die Aufgabe der jeweiligen Schriftleitung sein, unfaire Begutachtung zu vermeiden oder zu erkennen und dann abzulehnen.

Der Weg von Empfang eines Manuskripts bis zur Veröffentlichung ist ein langer. Nicht jeder Beitrag, selbst der bestreferierte, mag nicht angenommen werden, unabhängig von seiner Güte. Denn jede Zeitschrift hat ihr eigenes „Bild“, gemalt von vielen Händen, das mit der Zeit entstanden ist und dessen Thema wie ihre Farben die Perspektiven einer Schriftleitung wie auch die bestimmten Wünsche und Bedürfnisse des Leserkreises vereint.

Wie also sollte man nun heutzutage den „peer review“ beurteilen? Ist dieser Vorgang so gut und wichtig wie man es sich versprochen hatte? Es sei jedem überlassen, darüber für sich zu entscheiden. Man sollte sich aber an das Wappen des Prince of Wales erinnern, denn dort ist geschrieben: Honi soit qui mal y pense (Übel dem, der übel denkt). 